\title{
Coupling Vascular and Myocardial Inflammatory Injury into a Common Phenotype of Cardiovascular Dysfunction: Systemic Inflammation and Aging - A Mini-Review
}

\author{
Valentina O. Puntmann ${ }^{\mathrm{a}}$ Peter C. Taylor ${ }^{\mathrm{b}}$ Manuel Mayr ${ }^{\mathrm{c}}$ \\ ${ }^{a}$ Cardiovascular Section, Department of Experimental Medicine, Division of Investigative Sciences, and \\ b Kennedy Institute of Rheumatology, Imperial College London, and 'King's BHF Centre, King's College London, \\ London, UK
}

\section{Key Words}

Cardiovascular disease $\cdot$ Inflammation, myocardial • Imaging, integrated, noninvasive $\cdot$ Immunomics $•$ Proteomics

\begin{abstract}
The rising epidemic of cardiovascular (CV) disease is fuelled by obesity, hypertension and diabetes and, independently and cumulatively, by an aging population. Extensive research identified immunoinflammatory mechanisms as key drivers in the initiation and progression of the disease, from early asymptomatic stages of vascular and myocardial injury leading to the clinically manifest dysfunction and remodeling in advanced stages. Underlying processes include endothelial dysfunction and extracellular matrix restructuration leading to increased vascular stiffness, as well as myocardial remodeling with dilatation and wall thinning. In this, overproduction of tumor necrosis factor- $\alpha$, amongst others, contributes to generalized CV injury and dysfunction. Moreover, recent insights into the involvement of innate and adaptive immunity in atherosclerosis have shed light on many interesting parallels with chronic systemic inflammatory conditions, such as rheumatoid arthritis, with aggravated inflammation-induced vascular and myocardial injury. Besides, chronologic age has been identified as a potent, indepen-
\end{abstract}

dent risk for reduced CV capacity and a plethora of heart diseases, with other modifiable risk factors acting as accelerators. We discuss the available evidence and propose that characterization of inflammatory $C V$ responses might reveal a distinctive $\mathrm{CV}$ inflammatory phenotype. A comprehensive noninvasive bio-signature, comprising immunomic biomarkers and integrated noninvasive imaging, may serve as a potential tool in the early diagnosis and prognostication of CV risk.

Copyright $\odot 2010$ S. Karger AG, Basel

\section{Inflammation and Atherosclerosis}

Atherosclerosis/atherothrombosis is the major cause of the rising epidemics of cardiovascular (CV) disease. Current views regard atherosclerosis as a dynamic and progressive disease arising from the combination of hypercholesterolemia, oxidative stress injury, and disrupted hemorheology with chronic inflammation acting as the common denominator [1]. The endothelium, the inner lining of all blood vessels, maintains homeostasis through a balance of endothelium-derived factors. Disruption by inflammatory and traditional CV risk factors leaves the vasculature susceptible to atherogenesis, the process of forming plaques in arteries. Inflammatory mediators

\section{KARGER}

Fax +41613061234

E-Mail karger@karger.ch

www.karger.com
(C) 2010 S. Karger AG, Basel

0304-324X/11/0574-0295\$38.00/0

Accessible online at:

www.karger.com/ger
Dr. Valentina O. Puntmann, Cardiovascular Section

Department of Experimental Medicine, Division of Investigative Sciences Imperial College London, Hammersmith Campus, Burlington Danes Building 5th Floor, Du Cane Road, London W12 0NN (UK)

Tel. +44 207594 6825, Fax +44 207594 7393, E-Mail v.puntmann@imperial.ac.uk 
play a fundamental role in the initiation, progression, and eventual rupture of plaques. Tumor necrosis factor (TNF)- $\alpha$, in particular, is a key mediator of inflammation and vascular dysfunction and progression of atherosclerotic disease. TNF- $\alpha$ activates a proinflammatory gene expression profile in endothelial cells and promotes the adherence and monocyte migration into the subendothelial layer. At a molecular level, E-selectins mediate leukocyte rolling, chemokines lead to leukocyte activation, and intercellular adhesion molecule 1 and vascular cell adhesion molecule 1 contribute to leukocyte adhesion. In the vasculature, monocytes undergo transformation into macrophages that internalize modified lipoproteins, giving rise to foam cells. In parallel, upregulation of hemostatic proteins induces a highly procoagulative state of platelets, promoting release of their contents at the site of activated endothelium. The evolution of a fatty streak towards a complex atherosclerotic lesion is characterized by amassed oxidized low-density lipoproteins (LDLs) exerting toxic effects on macrophages and smooth muscle cells, leading to the formation of a necrotic core. Insidious neovascularization further promotes plaque growth, and rupture of these newly formed, fragile vessels may result in an acute expansion of the lesion. Reparative mechanisms include the migration and proliferation of smooth muscle cells resulting in increased collagen synthesis and the formation of a fibrous cap. However, the continuous release of proinflammatory cytokines and activation of proteolytic enzymes can weaken the fibrous cap and ultimately lead to plaque rupture precipitating clot formation. Acute thrombotic complications - atherothrombosis - may result in vessel occlusion with the restriction in blood flow triggering the manifestation of disease, i.e. heart attacks (myocardial infarction). In interventional cardiology, in-stent thrombosis mimics acute vessel occlusion linked with high morbidity and mortality [2]. In addition to thrombogenicity of the stent, lesion characteristics with high inflammatory load and perturbed healing capacity may owe to the pathophysiology of stent thrombosis.

Endothelial dysfunction is an early hallmark of vascular disease. It is broadly used to refer to several pathological conditions, including altered anticoagulant and anti-inflammatory properties of the endothelium, impaired modulation of vascular growth, and dysregulation of vascular remodeling [3]. However, in much of the literature this term has been honed to impairment of endothelium-dependent vasorelaxation due to the lesser relative availability of endothelium-derived relaxing factors, most importantly nitric oxide (NO). NO plays a pivotal role in the regulation of vascular tone and vasomotor function, and is generated by the conversion of the amino acid L-arginine to NO and L-citrulline by the enzyme NO synthase. Several human studies have shown that inflammation and traditional risk factors for atherosclerosis predispose to endothelial dysfunction by reducing $\mathrm{NO}$ bioavailability due to a variety of reasons [3-5]: (1) accelerated NO degradation by excess of reactive oxidant species, such as superoxide anion, peroxynitrite; (2) decreased generation by NO synthase due to the lack of cofactors, such as tetrahydrobiopterin, and (3) the NOmediated vasodilatory effect is simply outweighed by contracting factors, including endothelin-1 and angiotensin II.

Endothelial dysfunction, as assessed in terms of vasomotor dysfunction, can occur well before the structural manifestation of atherosclerosis and thus can serve as an independent predictor of future CV events. Increased arterial stiffness, as reflected in a greater pulse pressure and pulse wave velocity, has been associated with many CV risk factors, and raised inflammatory biomarkers, such as high C-reactive protein levels [6, 7]. Indeed, as evidence accumulates linking inflammatory processes to atherogenesis, markers of inflammatory activation and endothelial dysfunction provide useful information about a patient's risk and stage of developing $\mathrm{CV}$ disease.

\section{Communalities with Systemic Inflammatory Conditions}

Several lines of evidence support the notion that inflammatory mechanisms represent the key drivers to $\mathrm{CV}$ injury and dysfunction and systemic inflammatory/autoimmune diseases, such as rheumatoid arthritis (RA), feature many intriguing parallels [8-10]. Patients with RA are at increased risk of coronary heart disease, have an increased standardized morality ratio and shortened life expectancy by 3-18 years in comparison to the otherwise matched non-RA population. These observations are further supported by the greater prevalence of atherosclerotic burden in this population, evidenced by significantly increased carotid intima-media thickness and the higher incidence of unstable carotid plaques, in the absence of clinically evident CV disease [9]. Importantly, the presence of subclinical atherosclerosis correlated with a greater degree of inflammatory response and duration of RA disease. Moreover, the presence of vascular dysfunction has been well documented in RA patients with early and long-standing disease by intra-arterial vasore- 
activity studies, as well as noninvasively by means of flow-mediated dilation of brachial artery and increased arterial stiffness in otherwise CV symptom-free patients. Indeed, the concept of inflammatory-driven atherogenesis is derived from the resemblance of unstable coronary lesions to the appearance of inflammatory synovitis in RA with abundant presence of cytokines, such as TNF- $\alpha$, IL-1, IL-6, activated inflammatory infiltrate (in particular macrophages, mast and T cells), expression of adhesion molecules (intercellular adhesion molecule 1, vascular cell adhesion molecule 1, E-selectin), release of proteolytic enzymes (MMPs) and the process of neo-angiogenesis $[1,10,11]$. All play an important role in the process of articular injury and destruction, as well as in the development of unstable atheroma lesions. Additional specific mechanisms shared between the two conditions are increased reactivity against bacterial and human heat shock protein (HSP)60/65 [12, 13], and proliferation of the Tlymphocyte subtype with downregulation of CD28 epitope, the (CD4CD $28^{0} \mathrm{~T}$-cell), characterized by proinflammatory and aggressive tissue-damaging properties [13, 14]. Moreover, immunosuppressive intervention, such as T-cell depletion, in early inflammatory stages prevents development of arteriosclerotic lesions induced by immunization with HSP65 [15]. Taken together, the above evidence lends support to the role of chronic persistent inflammation as the 'common denominator' of both conditions.

\section{Inflammation and Myocardial Injury}

The interplay between the inflammation and myocardium led to generation of the so-called 'cytokine hypothesis', linking the TNF- $\alpha$ overexpression with myocardial injury, left ventricular (LV) dysfunction and cardiac cachexia [16]. Although the functional significance of TNF$\alpha$ in the failing heart remains uncertain, this cytokine can mimic many aspects of the heart failure phenotype and the role of TNF- $\alpha$ is central to the cytokine-induced myocardial injury and pathogenesis of LV dysfunction for the following reasons. First, plasma levels of this cytokine and its soluble receptors are elevated in patients with heart failure; those heart failure patients with raised TNF- $\alpha$ levels above the 90 th percentile $(>6.6 \mathrm{pg} / \mathrm{ml})$ have significantly raised overall morbidity and mortality. Next, TNF- $\alpha$ directly suppresses cardiac contractility and induces apoptosis of cardiac myocytes. Differential receptor signaling is the key to TNF-induced responses [17]: proinflammatory and proapoptotic effects through
TNF- $\alpha$-induced sustained activation of nuclear factor $\kappa B$ are dependent on TNF receptor 1 signaling. In contrast, activation of TNF receptor 2 appears to confer immunoregulatory and cardioprotective functions during acute stress against hypoxic and ischemic injury, but has no effect on cell motion. Idiopathic, autoimmune, and infectious forms of inflammatory cardiomyopathies share many similarities with the heart failure model of inflammatory myocardial injury [18]. The well-established adverse effects of TNF- $\alpha$ lead to LV dysfunction/remodeling, via complex processes involving changes in extracellular matrix, mitochondrial energetics, and distinctive genetic and epigenetic modifications, such as activation of the fetal gene program and post-translational modifications. Stimulated by cardiac injury, such as myocardial infarction, overexpression of TNF- $\alpha$ is capable of upregulating the activity of MMPs [16]. Provoking an intermediate loss of myocardial fibrillar collagen and mural realignment of myofibrillar bundles, this precedes structural remodeling by increased fibrillar collagen content, leading towards a thin-walled, stiff and dilated ventricle, a hallmark of the end-stage remodeling process. Recent evidence suggests that reperfusion contributes to myocardial injury after ischemia via mitochondrial dysfunction, whereby opening of a nonspecific high-conductance channel, termed the mitochondrial permeability-transition pore, leads to collapse of the membrane potential, the uncoupling of the respiratory chain, and the activation of apoptotic pathways [19]. Recent evidence indicates that acute application of cyclosporine, a mitochondrial permeability-transition pore inhibitor and a potent immunosuppressive agent, in patients presenting with acute myocardial infarction substantially reduces infarct size [20].

\section{Inflammation and Cardiovascular Remodeling in Aging}

Several lines of evidence support the notion that ageassociated changes in the CV system parallel those observed in subclinical CV inflammation and cachexia [21]. Aging is associated with decreased vascular distensibility and increased arterial stiffness, paralleled by increased levels of oxidative stress and low-grade inflammation [7]. Moreover, cardiac structural remodeling, increased LV wall thickness and accumulation of extracellular matrix lead to reduced cardiac output, decreased LV end-systolic pressure, fractional shortening, and reduced CV reserve. In general, aging cardiac myocytes have lesser 
compensatory capacity due to reduced expression of antioxidative enzymes, altered function of the mitochondrial respiratory chain due to reduced expression of vital oxidases such as cytochrome c, leading to electron leakage and oxidative damage [22]. The aging process is also characterized by increased stiffness and impaired relaxation, in line with reduced bioavailability of sarcoplasmic reticulum $\mathrm{Ca}^{2+}$-ATPase, deranged homeostasis of cytoskeletal proteins, and post-transcriptional switch towards slow contractile protein isoforms of myosin [21]. While there appears to be a distinct difference between aging and coronary atherosclerosis-induced cardiac fibrosis, these effects may well overlap. In addition, the greater atherosclerotic burden in this population is laden with a high incidence of acute $\mathrm{CV}$ events and is the major cause of mortality and disability in aged people [23]. Advanced age is a marker of poor prognosis; $75 \%$ of all myocardial infarction-related deaths occur after the age of 70 and the greater preexisting CV load of prior myocardial infarction and congestive heart failure [23]. After myocardial infarction, these patients are highly prone to develop heart failure through adverse LV remodeling, including greater infarct expansion, impaired infarct healing and, importantly, exaggerated and prolonged inflammatory response [21]. Aging may adversely affect LV remodeling leading to progressive LV dilatation through modification of the inflammatory response after myocardial infarction.

\section{Coupling of Vascular and Myocardial Inflammation into Cardiovascular Inflammatory Phenotype - The Role for Integrated Bio-Signature}

Characterization of CV inflammation is becoming increasingly important in the management of patients with CV disease as a whole. In addition to playing a causal role in vascular/myocardial injury and remodeling, inflammation can yield predictive and prognostic information of potential clinical utility. As understanding of underlying mechanisms has improved, a number of soluble mediators of inflammation, such as high-sensitivity C-reactive protein, raised TNF- $\alpha$, antibodies to oxidized lipoproteins, to bacterial/human HSPs and to infectious agents [11], as well as noninvasive assessments of atherosclerotic burden, endothelial function, aortic stiffness and myocardial function, have been suggested as tools of CV risk assessment [6, 24]. Yet, the development of $\mathrm{CV}$ biomarkers can be challenging for several reasons. $\mathrm{CV}$ inflammation as a generalized systemic disease is inherently so complex that a single biomarker is unlikely to capture most of the individual predisposition to develop CV disease or herald its sequelae. Despite its intricacy, a recently proposed triad of vulnerable systems - vulnerable plaque, vulnerable blood, and vulnerable myocardium - designate possibilities for biomarker discovery and development of new treatment targets within a window of opportunity, available for biomarkers to emerge and allow intervention. The recent advent of new technologies, multidisciplinary lifescience approaches and advances in imaging have increased recognition that $\mathrm{CV}$ inflammation arises from dynamic dysregulation of several gene regulatory networks, proteins, and metabolic alterations, reflected in complex pathophysiological perturbations. Leading to particular phenotypes of pathology, this is likely to be amenable to morphological characterization ex vivo, such as with proteomics and immunomics [25], and in vivo, such as with imaging [26]. Integrating molecular biology with clinical physiology may increase the potential to decipher the complex bio-signature in time for intervention.

\section{Proteomics and Atherosclerosis}

'-Omics' techniques contrast the study of complete systems (a holistic approach) with the study of individual elements (a reductionist approach). Unlike the genome, the transcriptome, proteome and metabolome vary. Whereas the genome can predict risk, the transcriptome, proteome and metabolome can also assess the current patient's status. Our recent proteomic study revealed that plaque immunoglobulins exist not only as free deposits in human atherosclerotic lesions but also within plaque microparticles [27]. Microparticles are small membrane blebs triggered by physical or chemical stress from the cell surface. Plaque microparticles are derived from leukocytes (about 50\%), erythrocytes, smooth muscle cells and endothelial cells, but not platelets. The latter are probably cleared by macrophages [28]. Interestingly, the intravesicular antibody load within atherosclerotic plaques was confined to a subpopulation of CD14+ microparticles. The immunoglobulins trapped within microparticles were still reactive despite their engulfment by plaque macrophages and their antigen specificity was clearly distinct from the antibodies circulating in plasma. These findings do not exclude a carryover of plasma antibodies into the microparticle preparations, but at least the capture of plasma antibod- 
ies within atherosclerotic plaques was highly specific. Interestingly, plaque microparticles expressed MHC class II together with potent co-stimulatory molecules $[27,29]$ on their surface and activated CD $4+$ T lymphocytes, which could contact and stimulate B lymphocytes, although scarce in atherosclerotic plaques, to produce immunoglobulins specific against plaque antigens. Thus, besides being an important determinant of plaque thrombogenicity, plaque microparticles might play a previously unrecognized role in modulating vascular inflammation and immunological profiling could contribute to a better understanding of the systemic versus local immune activation.

\section{Immunomics - Just One More '-Ome'?}

The new concept of 'immunomics' aims at measuring the immunoglobulin portfolio in individuals by exploiting combinatorial antigen libraries. This could be particularly important in the elderly. The immune system becomes less responsive as people age. Since the production of antibodies is impaired, a comprehensive assessment of the overall directionality/activation state of the patients' immune response may be informative for correctly interpreting the relevance of antibody levels against specific antigens. For example, correlations between antibodies might be confounded by the varying natural tendency of people to make antibodies in general. On the other hand, reactivity against a particular antigen may differ according to the immunoglobulin class, i.e. divergent findings between IgM and IgG autoantibodies to oxidized LDL in CV disease are increasingly being reported as investigators are more frequently measuring both subtypes in the same datasets.

In a recent study, we applied 'immunomic' methods to partially unravel the antigen specificity of immunoglobulins deposited within human atherosclerotic plaques [25]. This immunomic experiment revealed that apart from antibodies to oxidized lipoproteins [27], certain anticarbohydrate moiety antibodies were also enriched in atherosclerotic lesions. This became evident because the anticarbohydrate moiety antibody portfolio within plaques differed profoundly from the one in plasma of the same patients. Thus, the capture of plasma antibodies within atherosclerotic lesions must be highly specific. Among the antigens recognized within plaques were carbohydrate antigens of the $\mathrm{ABO}$ blood group and the antigen responsible for hyperacute rejection in xenotransplantation, the Gal- $\alpha-(1,3)-G a l$ linkage. This terminal carbohydrate epitope is the major target for natural antibodies to pig cells in humans and formed by the $\alpha-1,3$ galactosyl transferase, which places a terminal galactose residue in an $\alpha$-linkage to another galactose. Plaque antibodies against the blood group A antigen were detected in all but 1 patient who was blood group A positive. The anti-A antibodies were of 3 heavy chain isotypes: IgG2, $\operatorname{IgA}$, and IgM (fig. 1). The undetectable levels of IgD in atherosclerotic plaques may be attributed to proteolysis. IgD is more susceptible to proteolytic cleavage compared to IgG2, IgA or IgM and would have a shorter half-life in atherosclerotic plaques with high proteolytic activity. Blood group antigens are expressed at high levels on endothelium, probably attached to von Willebrand factor and the presence of anticarbohydrate antibodies within human atherosclerotic lesions extends previous observations that the pattern of the anticarbohydrate immune response is different in plasma from patients with advanced atherosclerosis [28]. Although our 'immunomic profiles' identified anticarbohydrate immunoglobulins as a component of the plaque antibodies present, our findings do not exclude other antibody specificities. For example, peptide antigens tend to be recognized as conformational rather than linear epitopes. In addition, lipids, modified lipid reactivities, and peptides with posttranslational modifications were not present in the combinatorial library. Additionally, these findings per se do not necessarily imply alterations in the amount or disposition of carbohydrate antigens but could just reflect alterations in the immune system affecting the levels of natural antibodies to common environmental antigens. On the other hand, a recent report demonstrated the presence of foreign sugars in subendothelial regions and in the endothelium overlying atherosclerotic plaques [30]: the common mammalian sialic acid N-glycolylneuraminic acid (Neu5Gc) is not expressed in humans, but Neu5Gc-rich foods, such as red meat and milk products, deliver this immunogenic sialic acid to endothelial cells. Our intracellular biochemical pathways cannot distinguish between Neu5Gc and its endogenous precursor resulting in incorporation of Neu5Gc at the outer end of glycan chains. An anti-Neu5Gc antibody response contributes to endothelial inflammation and is more pronounced for certain presentations of Neu5Gc, known to be upregulated on endothelial cells by TNF- $\alpha$ [31]. Moreover, conjugating a protein antigen to a foreign sugar can lead to enhanced production of antibodies against the protein itself, with the foreign glycan acting as an adjuvant. 
Fig. 1. Immunomics. Atherosclerotic plaque microparticles (pellet), microparticle-depleted plaque supernatant, and plasma were obtained from the same patient and screened against a GlycoPanel sublibrary, containing various carbohydrate antigens coated onto tiny aluminum particles with a unique barcode (Ultraplex, Pronostics, Cambridge, UK). The immunoreactivity of the samples was determined by labeling the bound antibodies with a fluorescent detection reagent in which different antibody subclasses were labeled with different colors. After mixing, the barcode of the particles with positive reactivity was read in a microscopebased reader, and the amount of label bound to each bead code was plotted providing an immunomic profile. IgG2, IgA, and IgM against the blood group A antigen are marked with arrows on the $\mathrm{x}$-axis of the immunologic profiles. Adapted with permission from Mayr et al. [25].

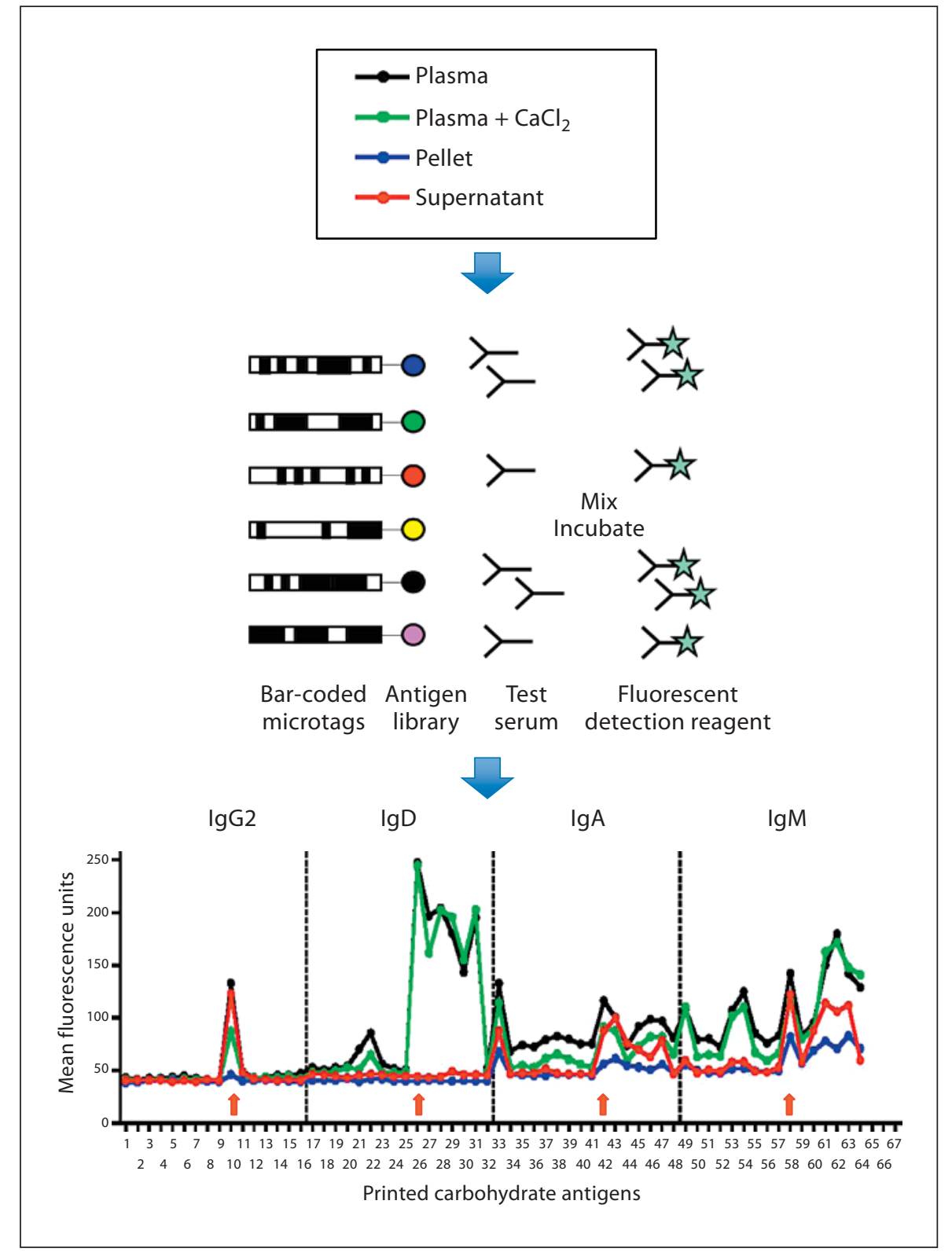

\section{Antigens in Atherosclerosis}

Heterophile antibodies - antibodies raised against an antigen from one species that also reacts against antigens from other species - have been implicated in atherogenesis: immunization experiments with Streptococcus pneumoniae were shown to induce autoantibodies to oxidized LDL, which proved to be antiatherogenic in animals [32]. The potential effects of unstimulated antioxidized LDL antibody levels on human atherosclerosis and clinical CV disease, however, seem to be less pronounced
[33]. Bacterial HSPs provide another example of antigenic mimicry, sharing a high sequence similarity with the mammalian homolog. Immunization experiments with mycobacterial HSP65 were proatherogenic $[32,34]$. In contrast, antiatherogenic effects were noted upon induction of immunological tolerance against HSP60/65 [35]. Given their phylogenetically conserved structure, bacterial HSPs appear to induce a cross-reactive immune response against inadvertent presentation of human HSPs on the surface of stressed endothelial cells. It is well recognized that traditional atherogenic stimuli act as endo- 
Fig. 2. Measurement of aortic stiffness by MRI is obtained by velocity encoded flowimaging in oblique transverse plane on the level of pulmonary artery, as indicated by the representative flow curves in the ascending aorta and descending aorta, plotted against the 45 imaging phases. The intersection of the tangent line to the upstroke and the baseline was considered as the arrival time of the pulse wave. $\mathrm{dT}$ denotes the time delay of the arrival of pulse wave at the two levels. A standardized approach is used to measure the path length between the middle of both levels ( $\mathrm{dS}$ ), as featured by the orange line following the midline course of the aorta on Proton density-weighted black-blood anatomical image of aortic 'candy cane'. Aortic pulse wave velocity $(\mathrm{PWV})$ is defined by $\mathrm{dS} / \mathrm{dT}$ $(\mathrm{m} / \mathrm{s})$.

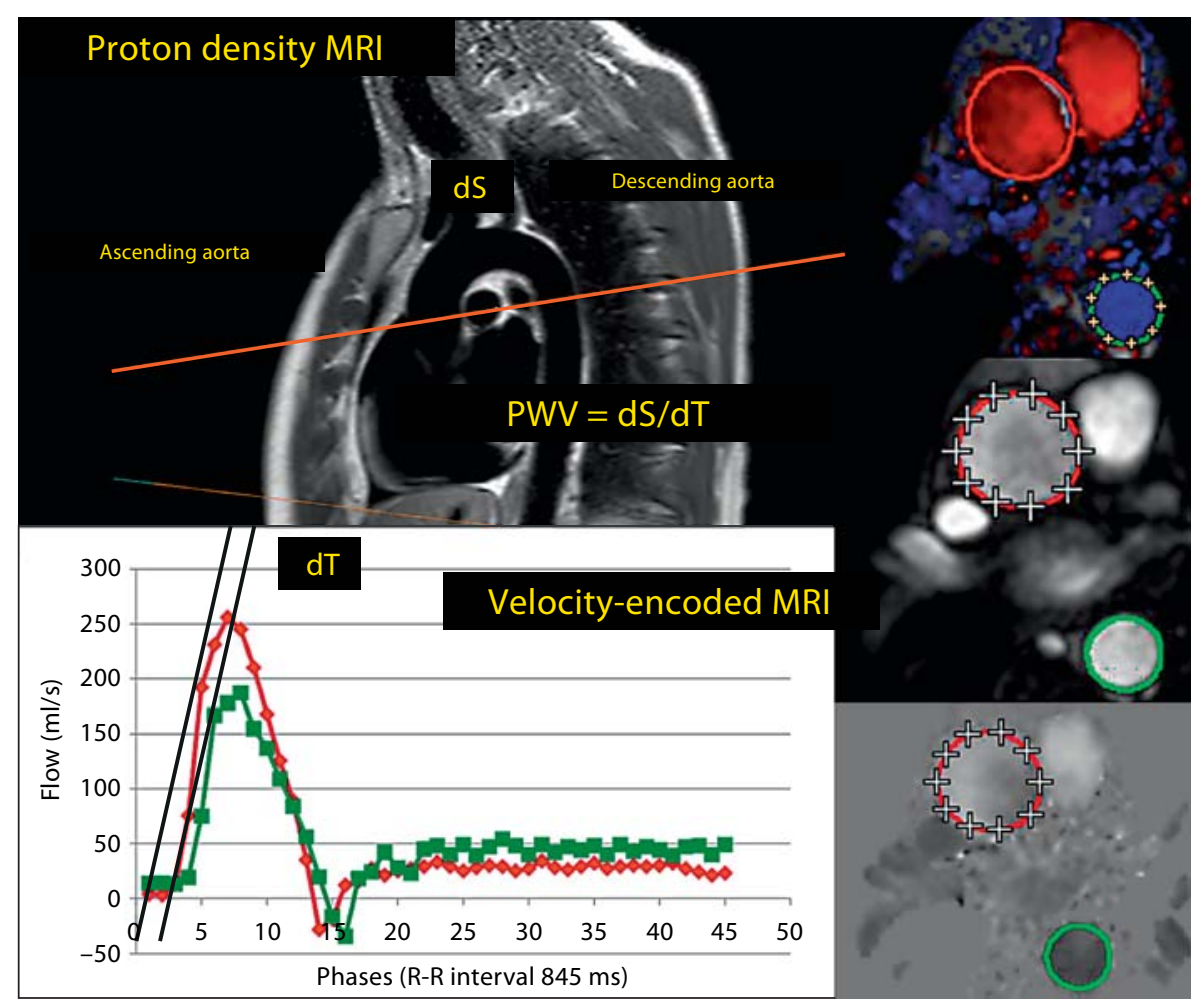

thelial stressors and human HSPs are expressed as part of a general stress response in the vasculature and recognized by antibacterial HSP antibodies [11-13]. Thus, the endothelial stress response may contribute to a translation of the influence of classical atherosclerosis risk factors into mounting an inflammatory response within the vascular wall. Levels of anti-HSP60/65 antibodies were correlated with atherosclerosis progression in most studies published to date [11] and correlated to seropositivity to infectious agents [12]. A recent report relating intensive periodontal treatment with an acute, short-term rise in systemic inflammatory markers and endothelial dysfunction further corroborates the role of bacteria as an additional driver in the inflammatory vascular response [36].

\section{The Role of Integrated Cardiovascular Imaging}

Integrating molecular biology with clinical physiology may increase the potential to decipher the complex biosignature in time for intervention. Leading to particular phenotypes of pathology, this is likely to be amenable to morphological characterization in vivo, such as with imaging. Many contemporary imaging techniques may prove their utility in offering a fast and noninvasive appreciation of these processes in vivo, ideally in the context of routinely applicable protocols and prior to development of overt clinical symptoms. In addition to the aforementioned examples of measuring carotid intima media thickness and assessment of endothelial dysfunction (fig. 2), detailed plaque characterization via intraluminal imaging is now possible, supporting the estimation of vascular instability and associated risk of events. Indeed, various imaging techniques contribute important information due to their inherent advantages: while imaging with magnetic resonance imaging (MRI) provides broad access to visualizing planes and detailed and morphological characterization with no radiation, computed tomographic coronary angiography may be able to provide fast and noninvasive luminal information once the issues around radiation exposure and resolution comparable to invasive coronary angiography are resolved. Noninvasive characterization of cardiac structure and function by cardiac MRI is instrumental for assessing the natural history of myocardium in areas affected by atherosclerotic/ atherothrombotic lesions. Integrated, multiparametric imaging of myocardial edema with T2-weighted sequences and scar imaging with late gadolinium enhancement reveals the differential myocardial phenotypes in 
the presence of self-limiting myocardial inflammation, such as in myocarditis, and sustained systemic inflammation in patients with RA [26]. Moreover, in patients with established coronary artery disease it helps to predict the recovery of myocardial function in dysfunctional segments after acute myocardial infarction and by surgical or percutaneous revascularization [37]. MRI thus plays an important role in selecting patients who are most likely to benefit from intervention. Intraluminal information is crucial and high-resolution methods include forward-looking adaptations of intravascular ultrasound (IVUS), optical coherence tomography, and intravascular MRI [38]. Many of these imaging modalities can be coupled with interventional techniques, and thus improve upon the guidance provided by angiography during revascularization. IVUS is a particularly appealing imaging modality for image guidance purposes due to its high resolution, reasonable penetration depth, and its ability to readily identify the external elastic lamina in the absence of calcifications [39]. IVUS-based techniques such as elastography, radiofrequency tissue characterization or virtual histology can be incorporated with modern IVUS systems to identify the mechanical properties and composition of atherosclerotic lesions. Potential future tools in CV imaging feature targeted contrast agents for vascular inflammation and plaque characterization, identifying expression of adhesion molecules, activation of MMPs or HSPs [40], or the early angiogenic expansion of the vasa vasorum that supports plaque development.
Until the point of clinical applicability, IVUS and optical coherence tomography continue to be important (but invasive) tools for plaque characterization, but ultimately the molecular imaging methodologies may allow direct quantification of the causative molecular constituents of disease and help to define a distinctive CV inflammatory phenotype.

\section{Conclusions}

Syndromes of increased systemic CV inflammation are characterized by concomitant vascular and myocardial injury, leading to dysfunction and remodeling. Available evidence of anti-inflammatory interventions in the context of systemic inflammatory diseases further corroborates the role of inflammation in initiation, progression and developments of its end-stage complications, such as atheroma formation and heart failure. 'Systems biology' of soluble biomarkers, coupled with 'systems physiology' by means of noninvasive imaging may advance our understanding of disease processes at a 'pathway' instead of at a 'single molecule' level. A comprehensive bio-signature of CV diseases could be exploited in the early diagnosis and prognostication but also prove its utility in drug discovery, pertinent in future assessments of any CV benefit/harm of existing and emerging therapies.

\section{References}

1 Libby P, Ridker PM, Hansson GK: Inflammation in atherosclerosis from pathophysiology to practice. J Am Coll Cardiol 2009;54: 2129-2138.

$>$ Lüscher TF, Steffel J, Eberli FR, Joner M, Nakazawa G, Tanner FC, Virmani R: Drugeluting stent and coronary thrombosis: biological mechanisms and clinical implications. Circulation 2007;115:1051-1058.

3 Cai H, Harrison DG: Endothelial dysfunction in cardiovascular diseases: the role of oxidant stress. Circ Res 2000;87:840-844.

-4 Püntmann VO, Hussain MB, Mayr M, Xu Q, Singer DR: Role of oxidative stress in angiotensin-II mediated contraction of human conduit arteries in patients with cardiovascular disease. Vascul Pharmacol 2005;43: 277-282.
5 Hussain MB, Puntmann VO, Mayr M, Khong T, Singer DRJ: The role of oxidant stress in angiotensin II-mediated contraction of human resistance arteries in the state of health and the presence of cardiovascular disease. Vascul Pharmacol 2006;45:395-399.

6 Mitchell GF: Arterial stiffness and wave reflection: biomarkers of cardiovascular risk. Artery Res 2009;3:56-64.

7 Mattace-Raso FUS, van der Cammen TJM, van der Meer IM, Schalekamp MADH, Asmar R, Hofman A, Witteman JCM: C-reactive protein and arterial stiffness in older adults: the Rotterdam Study. Atherosclerosis 2004;176:111-116.

8 Van Leuven SI, Franssen R, Kastelein JJ, Levi M, Stroes ES, Tak PP: Systemic inflammation as a risk factor for atherothrombosis. Rheumatology 2008;47:3-7.
-9 Gonzalez-Gay MA, Gonzalez-Juanatey C, Pineiro A, Garcia-Porrua C, Testa A, Llorca $\mathrm{J}$ : High-grade C-reactive protein elevation correlates with accelerated atherogenesis in patients with rheumatoid arthritis. J Rheumatol 2005;32:1219-1223.

10 Taylor PC, Sivakumar B: Hypoxia and angiogenesis in rheumatoid arthritis. Curr Opin Rheumatol 2005; 17:293-298.

11 Wick G, Knoflach M, Xu Q: Autoimmune and inflammatory mechanisms in atherosclerosis. Annu Rev Immunol 2004;22:361403.

$12 \mathrm{Xu} \mathrm{Q}$, Willeit J, Marosi M, Kleindienst R, Oberhollenzer F, Kiechl S, Stulnig T, Luef G, Wick G: Association of serum antibodies to heat-shock protein 65 with carotid atherosclerosis. Lancet 1993;341:255-325. 
13 Mayr M, Kiechl S, Willeit J, Wick G, Xu Q: Infections, immunity, and atherosclerosis: associations of antibodies to Chlamydia pneumoniae, Helicobacter pylori, and cytomegalovirus with immune reactions to heatshock protein 60 and carotid or femoral atherosclerosis. Circulation 2000;102:833-839.

- 14 Liuzzo G, Kopecky SL, Frye RL, O’Fallon WM, Maseri A, Goronzy JJ, Weyand CM: Perturbation of the T-cell repertoire in patients with unstable angina. Circulation 1999; 100:2135-2139.

15 Metzler B, Mayr M, Dietrich H, Singh M, Wiebe E, Xu Q, Wick G: Inhibition of arteriosclerosis by $\mathrm{T}$-cell depletion in normocholesterolemic rabbits immunized with heat shock protein 65 . Arterioscler Thromb Vasc Biol 1999;19:1905-1911.

16 Mann DL: Inflammatory mediators and the failing heart: past, present and foreseeable future. Circ Res 2002;91:988-998.

$\checkmark 17$ Hamid T, Gu Y, Ortines RV, Bhattacharya C, Wang G, Xuan YT, Prabhu SD: Divergent tumor necrosis factor receptor-related remodeling responses in heart failure: role of nuclear factor-kappaB and inflammatory activation. Circulation 2009;119:1386-1397.

18 Pauschinger M, Chandrasekharan K, Schultheiss HP: Myocardial remodeling in viral heart disease: possible interactions between inflammatory mediators and MMP-TIMP system. Heart Fail Rev 2004;9:21-31.

-19 Currie RW, Tanguay RM, Kingma JG Jr: Heat-shock response and limitation of tissue necrosis during occlusion/reperfusion in rabbit hearts. Circulation 1993;87:963-971.

20 Piot C, Croisille P, Staat P, Thibault H, Rioufol G, Mewton N, Elbelghiti R, Cung TT, Bonnefoy E, Angoulvant D, Macia C, Raczka F, Sportouch C, Gahide G, Finet G, AndréFouët X, Revel D, Kirkorian G, Monassier JP, Derumeaux G, Ovize M: Effect of cyclosporine on reperfusion injury in acute myocardial infarction. N Engl J Med 2008;359:473481.

-21 Bernhard D, Laufer G: The aging cardiomyocyte: a mini-review. Gerontology 2008;54: 24-31.

-22 Li SY, Du M, Dolence EK, Fang CX, Mayer GE, Ceylan-Isik AF, LaCour KH, Yang X, Wilbert CJ, Sreejayan N, Ren J: Aging induces cardiac diastolic dysfunction, oxidative stress, accumulation of advanced glycation endproducts and protein modification. $\mathrm{Ag}$ ing Cell 2005;4:57-64.
23 Barakat K, Wilkinson P, Deaner A, Fluck D, Ranjadayalan K, Timmis A: How should age affect management of acute myocardial infarction? A prospective cohort study. Lancet 1999;353:955-959.

24 Puntmann VO: How-to guide on biomarkers: biomarker definitions, validation and applications with examples from cardiovascular disease. Postgrad Med J 2009;85:538545.

25 Mayr M, Grainger D, Mayr U, Leroyer AS, Leseche G, Sidibe A, Herbin O, Yin X, Gomes A, Madhu B, John R: Griffiths JR, Xu Q, Tedgui $\mathrm{A}$ and Boulanger CM: Proteomics, metabolomics, and immunomics on microparticles derived from human atherosclerotic plaques. Circ Cardiovasc Genet 2009;2: 379-388.

26 Puntmann VO, Taylor PC, Barr A, Schnackenburg B, Jahnke C, Paetsch I: Towards understanding the phenotypes of myocardial involvement in the presence of self-limiting and sustained systemic inflammation: a magnetic resonance imaging study. Rheumatology 2010;49:528-535.

27 Mosedale DE, Chauhan A, Schofield PM, Grainger DJ: A pattern of anti-carbohydrate antibody responses present in patients with advanced atherosclerosis. J Immunol Methods 2006;309:182-191.

28 Prokopi M, Pula G, Mayr U, Devue C, Gallagher J, Xiao Q, Boulanger CM, Westwood N, Urbich C, Willeit J, Steiner M, Breuss J, Xu Q, Kiechl S, Mayr M: Proteomic analysis reveals presence of platelet microparticles in endothelial progenitor cell cultures. Blood 2009; 114:723-732.

29 Xu QB, Oberhuber G, Gruschwitz M, Wick G: Immunology of atherosclerosis: cellular composition and major histocompatibility complex class II antigen expression in aortic intima, fatty streaks, and atherosclerotic plaques in young and aged human specimens. Clin Immunol Immunopathol 1990; 56:344-359.

30 Pham T, Gregg CJ, Karp F, Chow R, PadlerKaravani V, Cao H, Chen X, Witztum JL, Varki NM, Varki A: Evidence for a novel human-specific xeno-auto-antibody response against vascular endothelium. Blood 2009; 114:5225-5235.

31 Padler-Karavani V, Yu H, Cao H, Chokhawala H, Karp F, Varki N, Chen X, Varki A: Diversity in specificity, abundance, and composition of anti-Neu5Gc antibodies in normal humans: potential implications for disease. Glycobiology 2008;18:818-830.

- 32 Binder CJ, Hörkkö S, Dewan A, Chang MK, Kieu EP, Goodyear CS, Shaw PX, Palinski W, Witztum JL, Silverman GJ: Pneumococcal vaccination decreases atherosclerotic lesion formation: molecular mimicry between Streptococcus pneumoniae and oxidized LDL. Nat Med 2003;9:736-743.
33 Mayr M, Kiechl S, Tsimikas S, Miller E, Sheldon J, Willeit J, Witztum JL, Xu Q: Oxidized low-density lipoprotein autoantibodies, chronic infections, and carotid atherosclerosis in a population-based study. J Am Coll Cardiol 2006;47:2436-2443.

$34 \mathrm{Xu}$ Q, Dietrich H, Steiner HJ, Gown AM Schoel B, Mikuz G, Kaufmann SH, Wick G: Induction of arteriosclerosis in normocholesterolemic rabbits by immunization with heat shock protein 65 . Arterioscler Thromb 1992;12:789-799.

35 Maron R, Sukhova G, Faria AM, Hoffmann E, Mach F, Libby P, Weiner HL: Mucosal administration of heat shock protein-65 decreases atherosclerosis and inflammation in aortic arch of low-density lipoprotein receptor-deficient mice. Circulation 2002;106: 1708-1715.

- 36 Tonetti MS, D’Aiuto F, Nibali L, Donald A, Storry C, Parkar M, Suvan J, Hingorani AD, Vallance P, Deanfield J: Treatment of periodontitis and endothelial function. N Engl J Med 2007;356:911-920.

-37 Klein C, Nagel E, Gebker R, Kelle S, Schnackenburg B, Graf K, Dreysse S, Fleck E: Magnetic resonance adenosine perfusion imaging in patients after coronary artery bypass graft surgery. JACC Cardiovasc Imaging 2009;2:437-445.

38 Von Birgelen C, Hartmann M, Mintz GS, van Houwelingen $K G$, Deppermann $N$, Schmermund A, Bose D, Eggebrecht H, Neumann T, Gossl M, Wieneke H, Erbel R: Relationship between cardiovascular risk as predicted by established risk scores versus plaque progression as measured by serial intravascular ultrasound in left main coronary arteries. Circulation 2004;110:1579-1585.

-39 Rieber J, Meissner O, Babaryka G, Reim S, Oswald M, Koenig A, Schiele TM, Shapiro M, Theisen K, Reiser MF, Klauss V, Hoffmann U: Diagnostic accuracy of optical coherence tomography and intravascular ultrasound for the detection and characterization of atherosclerotic plaque composition in ex-vivo coronary specimens: a comparison with histology. Coron Artery Dis 2006; 17:425-430.

-40 Wick MC, Kremser C, Frischauf S, Wick G: In vivo molecular imaging of vascular stress. Cell Stress Chaperones 2008;13:263-273. 